\title{
DELTA EXCITATION IN DEUTERON-PROTON ELASTIC SCATTERING
}

\author{
N.B.Ladygina ${ }^{1 \mathrm{a}}$ \\ Joint Institute for Nuclear Research, LHEP, Dubna, Russia \\ Received: date / Revised version: date
}

\begin{abstract}
Deuteron-proton elastic scattering is studied in the multiple scattering expansion formalism. The four contributions are taken into account: one-nucleon-exchange, single- and double scattering, and $\Delta$ isobar excitation. The presented approach was applied to describe the differential cross sections at deuteron energies between 500 and $1300 \mathrm{MeV}$ in a whole angular range. The obtained results are compared with the experimental data.
\end{abstract}

PACS. 21.45.+v Few-body systems - 25.45.-z 2H-induced reactions - 25.45.De Elastic and inelastic scattering $-24.10 . \mathrm{Jv}$ Relativistic models

\section{Introduction}

In recent years heavy ion physics at high energies has been very popular. There are many efforts to study some systems containing a lot of nucleons. However, the energy in such reactions is quite large to appear the multiple nucleon scattering. Therefore, the problem of the nucleon-nucleon interaction is topical up to now. Even at low energies all the theoretical calculations are based on the phenomenological nucleon-nucleon potential. The problem becomes more difficult when the energy is enough for a manifestation of inelastic channels.

Elastic deuteron-proton scattering is the simplest example of the hadron nucleus collision, because a deuteron is the simplest nucleus containing only one proton and one neutron. The study of the deuteron-proton elastic scattering has a longtime story. The first nucleon-deuteron experiments were performed already in fifties of the previous century [1-[7]. Differential cross sections [1-[4] and polarization [5- 7 ] were measured at a few hundred $\mathrm{MeV}$. This topic has been intensively investigated in the 1970's and 80 's $8-[15$. Nowadays this reaction is still the subject of the investigations [16-20].

In the previous papers 21, 22, we considered this reaction in the multiple scattering expansion formalism. Three contributions, one-nucleon-exchange (ONE), single- scattering, and double scattering, were taken into account. We got a reasonable agreement for the differential cross sections between the theoretical predictions and the experimental data almost in a whole angular range. However, the rise of the differential cross sections at the scattering angle larger than $140^{\circ}$ was not described in the approach.

Send offprint requests to:

a Present address: nladygina@jinr.ru
In 1969 A.Kerman and L.Kisslinger supposed that resonances can play an important role in the deuteron-proton backward elastic scattering [23]. They suggested that a deuteron contains the $N^{*}$ resonance with $0.5-1 \%$ probability. However, an analysis of the experimental data using the Kerman- Kisslinger model [9] has demonstrated strong dependence of the obtained results on a deuteron wave function.

Later the double-scattering mechanism with $\Delta$-isobar in the intermediate state was taken into account in the dp backward scattering. The significant contribution of this diagram to the reaction was shown in refs. [24]- 26]. However, the double scattering with nucleon in an intermediate state was not considered in these papers . Perhaps, it was the reason why the description of the differential cross sections energy dependence was not good enough.

The effort to take the $\Delta$-isobar into account in order to describe dp-elastic scattering was also done in [27, 28. [17, where the $\Delta$-isobar term was included into the CD Bonn potential. In these papers deuteron-proton scattering was considered in a whole angular range, not only at $\theta^{*}=180^{\circ}$. Unfortunately, the process was studied at low energies, $T_{p} \leq 250 \mathrm{MeV}$, where the $\Delta$-isobar effects are negligible.

In this paper we keep on considering deuteron-proton elastic scattering in the framework offered in papers 21, [22. Here we add the $\Delta$-isobar contribution in our approach. The next part is devoted to the description of the general theoretical model. We briefly give the basic points of the multiple scattering approach. In section 3 the $\Delta$-excitation term is considered in a detail. Also the kinematics of the double scattering is given, both for the delta and nucleon in the intermediate state. In the last section we discuss the obtained results and compare them with the experimental data. 


\section{General formalism}

According to the three-body collision theory, the amplitude of deuteron-proton elastic scattering $\mathcal{J}$ is defined by the matrix element of the transition operator $U_{11}$ :

$$
\begin{aligned}
U_{d p \rightarrow d p}= & \delta\left(E_{d}+E_{p}-E_{d}^{\prime}-E_{p}^{\prime}\right) \mathcal{J}= \\
& <1(23)\left|\left[1-P_{12}-P_{13}\right] U_{11}\right| 1(23)>.
\end{aligned}
$$

Here the state $\mid 1(23)>$ corresponds to the configuration, when nucleons 2 and 3 form the deuteron state and nucleon 1 is free. Appearance of the permutation operators for two nucleons $P_{i j}$ reflects the fact that the initial and final states are antisymmetric due to an exchange of the two particles.

Following the Alt-Grassberger-Sandhas formalism 29, [30. we write the transition operator $U_{\beta \alpha}$ as:

$$
U_{\beta \alpha}=-\left(1-\delta_{\beta \alpha}\right)\left(H_{0}-z\right)-\sum_{\delta \neq \alpha} U_{\beta \delta} g_{0} t_{\delta} .
$$

This transition operator depends on the potential through the channel transition-operator $t_{\alpha}$ of the two-particle subsystems:

$$
\begin{aligned}
& t_{\alpha}=V_{\alpha}-V_{\alpha} g_{\alpha} V_{\alpha} \\
& t_{\alpha}=V_{\alpha}-V_{\alpha} g_{0} t_{\alpha},
\end{aligned}
$$

where $g_{0}$ is a free three-particle propagator and $g_{\alpha}$ is a two-particle resolvent in the three-particle space. In such a way we get the following equations for the transition operators with the rearrangement:

$$
\begin{aligned}
& U_{11}=t_{2} g_{0} U_{21}+t_{3} g_{0} U_{31} \\
& U_{21}=g_{0}^{-1}+t_{1} g_{0} U_{11}+t_{3} g_{0} U_{31} \\
& U_{31}=g_{0}^{-1}+t_{1} g_{0} U_{11}+t_{2} g_{0} U_{21},
\end{aligned}
$$

where $t_{1}=t(2,3)$,etc., is a t-matrix of the two-particle interaction. The indices $i j$ for the transition operators $U_{i j}$ denote free particles $i$ and $j$ in the final and initial states, respectively.

Iterating these equations up to the second order terms of $t_{i}$ we can present the reaction amplitude as a sum of the following four contributions: one nucleon exchange, single scattering and double scattering with the nucleon and delta in the intermediate state, -

$$
\mathcal{J}_{d p \rightarrow d p}=\mathcal{J}_{O N E}+\mathcal{J}_{S S}+\mathcal{J}_{D S}+\mathcal{J}_{\Delta}
$$

The first contribution into the $d p$-elastic scattering amplitude $\mathcal{J}$ in Eq.(5) is the one nucleon exchange (ONE) term.

$$
\mathcal{J}_{O N E}=-2<1(23)\left|P_{12} g_{0}^{-1}\right| 1(23)>
$$

The corresponding diagram is presented in Fig.1a. Applying the definitions of the wave function of a moving deuteron and three- nucleon free propagator, we can write ONE amplitude in the following form:

$$
\begin{aligned}
\mathcal{J}_{O N E}= & -2_{1(23)}<\boldsymbol{p}^{\prime} m^{\prime} \tau^{\prime} ;-\boldsymbol{P}_{d} \mathcal{M}_{d}^{\prime} 0 \mid \Omega_{d}^{\dagger}(23) P_{12} \\
& \left(E_{d}+E_{p}-\hat{K}_{1}-\hat{K}_{2}-\hat{K}_{3}+i \varepsilon\right) \\
& \Omega_{d}(23) \mid \boldsymbol{P}_{d} \mathcal{M}_{d} 0 ; \boldsymbol{p} m \tau>_{1(23)},
\end{aligned}
$$

where $m, m^{\prime}$ are spin projections of the initial and final protons, $\tau, \tau^{\prime}$ are their isospin projections, respectively. The kinetic-energy operator has a standard definition, $\hat{K}_{i}\left|\boldsymbol{p}_{i}>=\sqrt{m_{N}^{2}+\boldsymbol{p}_{i}^{2}}\right| \boldsymbol{p}_{i}>$.

After a straightforward calculation we have the expression for the ONE amplitude

$$
\begin{aligned}
& \mathcal{J}_{O N E}=-\frac{1}{2}\left(E_{d}-E_{p}-\sqrt{m_{N}^{2}+\boldsymbol{p}^{2}-\boldsymbol{P}_{d}^{2}}\right) \\
& <\boldsymbol{p}^{\prime} m^{\prime} ;-\boldsymbol{P}_{d} \mathcal{M}_{d}^{\prime} \mid \Omega_{d}^{\dagger}(23) \\
& {\left[1+\left(\boldsymbol{\sigma}_{\mathbf{1}} \boldsymbol{\sigma}_{\mathbf{2}}\right)\right] \Omega_{d}(23) \mid \boldsymbol{P}_{d} \mathcal{M}_{d} ; \boldsymbol{p} m>,}
\end{aligned}
$$

where the definition of the permutation operator in spin space $P_{12}(\sigma)=\frac{1}{2}\left[1+\left(\boldsymbol{\sigma}_{\mathbf{1}} \boldsymbol{\sigma}_{\mathbf{2}}\right)\right]$ has been applied.

All the calculations are performed in the deuteron Breit frame, where the deuterons move in opposite directions with equal momenta (Fig.1). It allows us to minimize the relative momenta of the nucleons in both deuterons. As a consequence, the non-relativistic deuteron wave function can be applied in the energy range under consideration.

In the rest frame the non-relativistic wave function of the deuteron depends only on one variable $\boldsymbol{p}_{0}$, which is the relative momentum of the outgoing proton and neutron:

$$
\begin{aligned}
<\mu_{p} \mu_{n}\left|\Omega_{d}\right| \mathcal{M}_{d}>=\frac{1}{\sqrt{4 \pi}}<\mu_{p} \mu_{n} \mid\left\{u\left(p_{0}\right)+\right. \\
\left.\frac{w\left(p_{0}\right)}{\sqrt{8}}\left[3\left(\boldsymbol{\sigma}_{\mathbf{1}} \hat{p}_{0}\right)\left(\boldsymbol{\sigma}_{\mathbf{2}} \hat{p}_{0}\right)-\left(\boldsymbol{\sigma}_{\mathbf{1}} \boldsymbol{\sigma}_{\mathbf{2}}\right)\right]\right\} \mid \mathcal{M}_{d}>
\end{aligned}
$$

where $u\left(p_{0}\right)$ and $w\left(p_{0}\right)$ describe $S$ and $D$ components of the deuteron wave function [31, 32, 33, $\hat{p}_{0}$ is a unit vector in $\boldsymbol{p}_{0}$ direction.

In order to get the wave function of the moving deuteron, it is necessary to apply the Lorenz transformations for the kinematical variables and Wigner rotations for the spin states. This procedure has been expounded in a detail in ref. 21]. The proton-neutron relative momenta for the initial $\boldsymbol{p}_{0}$ and final $\boldsymbol{p}_{0}^{\prime}$ deuterons are expressed in relativistic kinematics as:

$$
\begin{aligned}
& \boldsymbol{p}_{0}=\boldsymbol{p}+\boldsymbol{P}_{d}\left[1+\frac{E_{n}+E^{*}}{E_{p}+E_{n}+E^{*}}\right] \\
& \boldsymbol{p}_{0}^{\prime}=\boldsymbol{p}+\boldsymbol{P}_{d}\left[1-\frac{E_{n}+E^{*}}{E_{p}+E_{n}+E^{*}}\right] .
\end{aligned}
$$

Here $E_{n}=\sqrt{m_{N}^{2}+\boldsymbol{p}^{2}-\boldsymbol{P}_{d}^{2}}$ and $E^{*}=\sqrt{\left(E_{p}+E_{n}\right)^{2}-\boldsymbol{P}_{d}^{2}} / 2$ are the struck neutron energy in the moving deuteron frame and rest deuteron frame, respectively. Note, that $\left|\boldsymbol{p}_{0}\right|=\left|\boldsymbol{p}_{0}^{\prime}\right|$.

The other term in the $d p$-elastic scattering amplitude Eq.(5) is the single scattering (SS) one.

$$
\mathcal{J}_{S S}=2<1(23)\left|\left[1-P_{12}\right] t_{3}\right| 1(23)>
$$

The corresponding diagram is presented in Fig.(1b). Following a standard procedure we get the expression for the single scattering amplitude:

$$
\mathcal{J}_{S S}=\int d \boldsymbol{p}_{2}<-\boldsymbol{P}_{d} \mathcal{M}_{d}^{\prime}\left|\Omega_{d}^{\dagger}\right| \boldsymbol{p}_{2} m^{\prime \prime},-\boldsymbol{P}_{d}-\boldsymbol{p}_{2} m_{3}^{\prime}>
$$




$$
\begin{gathered}
<\boldsymbol{p}^{\prime} m^{\prime},-\boldsymbol{P}_{d}-\boldsymbol{p}_{2}\left|\frac{3}{2} t_{12}^{1}+\frac{1}{2} t_{12}^{0}\right| \boldsymbol{p} m, \boldsymbol{P}_{d}-\boldsymbol{p}_{2} m_{2}^{\prime}> \\
<\boldsymbol{p}_{2} m^{\prime \prime}, \boldsymbol{P}_{d}-\boldsymbol{p}_{2} m_{2}^{\prime}\left|\Omega_{d}\right| \boldsymbol{P}_{d} \mathcal{M}_{d}>
\end{gathered}
$$

The relative momenta of the two nucleons for the initial and final deuterons are

$$
\begin{aligned}
& \boldsymbol{p}_{0}=\boldsymbol{p}_{2}-\boldsymbol{P}_{d} \frac{E_{2}+E^{*}}{E_{2}+E_{3}+2 E^{*}} \\
& \boldsymbol{p}_{0}^{\prime}=\boldsymbol{p}_{2}+\boldsymbol{P}_{d} \frac{E_{2}+E^{\prime *}}{E_{2}+E_{3}^{\prime}+2 E^{\prime *}},
\end{aligned}
$$

where nucleons energies $E_{2}, E_{3}, E_{3}^{\prime}$ in the reference frame are defined by a standard manner (Fig.1b)

$$
\begin{aligned}
& E_{2}=\sqrt{m_{N}^{2}+\boldsymbol{p}_{2}^{2}}, \quad E_{3}=\sqrt{m_{N}^{2}+\left(\boldsymbol{P}_{d}-\boldsymbol{p}_{2}\right)^{2}} \\
& E_{3}^{\prime}=\sqrt{m_{N}^{2}+\left(\boldsymbol{P}_{d}+\boldsymbol{p}_{2}\right)^{2}}
\end{aligned}
$$

and these energies in the centre-of-mass of the two nucleons forming the initial and final deuterons are equal, correspondingly, to

$$
\begin{aligned}
& E^{*}=\frac{1}{2} \sqrt{\left(E_{2}+E_{3}\right)^{2}-\boldsymbol{P}_{d}^{2}}, \\
& E^{* *}=\frac{1}{2} \sqrt{\left(E_{2}+E_{3}^{\prime}\right)^{2}-\boldsymbol{P}_{d}^{2}} .
\end{aligned}
$$

The nucleon-nucleon scattering is described by the $t$ matrix element. We use the parameterization of this matrix offered by Love and Franey [34]. This is the on-shell $\mathrm{NN}$ t-matrix defined in the cente-of-mass:

$$
\begin{aligned}
& <\varkappa^{* \prime} \mu_{1}^{\prime} \mu_{2}^{\prime}\left|t_{c . m .}\right| \varkappa^{*} \mu_{1} \mu_{2}>=<\varkappa^{* \prime} \mu_{1}^{\prime} \mu_{2}^{\prime} \mid A+ \\
& B\left(\boldsymbol{\sigma}_{\mathbf{1}} \hat{N}^{*}\right)\left(\boldsymbol{\sigma}_{\mathbf{2}} \hat{N}^{*}\right)+C\left(\boldsymbol{\sigma}_{\mathbf{1}}+\boldsymbol{\sigma}_{\mathbf{2}}\right) \cdot \hat{N}^{*}+ \\
& D\left(\boldsymbol{\sigma}_{\mathbf{1}} \hat{q}^{*}\right)\left(\boldsymbol{\sigma}_{\mathbf{2}} \hat{q}^{*}\right)+F\left(\boldsymbol{\sigma}_{\mathbf{1}} \hat{Q}^{*}\right)\left(\boldsymbol{\sigma}_{\mathbf{2}} \hat{Q}^{*}\right) \mid \varkappa^{*} \mu_{1} \mu_{2}>.
\end{aligned}
$$

The orthonormal basis $\left\{\hat{q}^{*}, \hat{Q}^{*}, \hat{N}^{*}\right\}$ is a combination of the nucleon relative momenta in the initial $\varkappa^{*}$ and final $\varkappa^{\prime *}$ states:

$$
\hat{q}^{*}=\frac{\varkappa^{*}-\varkappa^{* \prime}}{\left|\varkappa^{*}-\varkappa^{* \prime}\right|}, \quad \hat{Q}^{*}=\frac{\varkappa^{*}+\varkappa^{* \prime}}{\left|\varkappa^{*}+\varkappa^{* \prime}\right|}, \quad \hat{N}^{*}=\frac{\varkappa^{*} \times \varkappa^{* \prime}}{\left|\varkappa^{*} \times \varkappa^{* \prime}\right|} .
$$

The amplitudes $A, B, C, D, F$ are the functions of the centreof-mass energy and scattering angle. The radial parts of these amplitudes are taken as a sum of Yukawa terms. A new fit of the model parameters 35] was done in accordance with the phase-shift-analysis data SP07 36].

Since the matrix elements are expressed via the effective $N N$-interaction operators sandwiched between the initial and final plane-wave states, this construction can be extended to the off-shell case allowing the initial and final states to get the current values of $\varkappa$ and $\varkappa^{\prime}$. Obviously, this extrapolation does not change the general spin structure.

The double scattering term can be divided into two parts: rescattering with a nucleon and $\Delta$-isobar in the intermediate state.

$$
\begin{aligned}
& \mathcal{J}_{D S}+\mathcal{J}_{\Delta}=2<N(1)\left|<\psi_{d}(23)\right| N(2) N(3)> \\
& <N(2) N(3)\left|\left[1-P_{12}\right]\right| t_{3} \mid\{N(1) N(2)+\Delta(1) N(2)\}> \\
& g_{0}<\{N(1) N(2)+\Delta(1) N(2)\}\left|t_{2}\left[1-P_{13}\right]\right| \\
& <N(2) N(3) \psi_{d}(23)>\mid N(1)>
\end{aligned}
$$

The double scattering contribution with the intermediate nucleon (DS) is defined by a deuteron wave function and two nucleon-nucleon t-matrixes. Also we have, here, three-nucleon propagator:

$$
\begin{aligned}
& \mathcal{J}_{D S}=\int d \boldsymbol{p}_{\mathbf{2}} d \boldsymbol{p}_{\mathbf{3}}^{\prime}<-\boldsymbol{P}_{d} \mathcal{M}_{d}^{\prime} \mid \Omega_{d}^{\dagger} \\
& \mid-\boldsymbol{P}_{d}-\boldsymbol{p}_{3}^{\prime} m_{2}^{\prime}, \boldsymbol{p}_{3}^{\prime} m_{3}^{\prime}> \\
& <\boldsymbol{p}^{\prime} m^{\prime},-\boldsymbol{P}_{d}-\boldsymbol{p}_{3}^{\prime} m_{2}^{\prime}, \boldsymbol{p}_{3}^{\prime} m_{3}^{\prime} \mid\left\{t_{3(N N)}^{1}\left(E^{\prime}\right) t_{2(N N)}^{1}(E)+\right. \\
& \left.\left[t_{3(N N)}^{1}\left(E^{\prime}\right)+t_{3(N N)}^{0}\left(E^{\prime}\right)\right]\left[t_{2(N N)}^{1}(E)+t_{2(N N)}^{0}(E)\right] / 4\right\} \\
& \left\{E_{d}+E_{p}-E_{1}-E_{2}-E_{3}^{\prime}+i \varepsilon\right\}^{-1} \\
& \mid \boldsymbol{p} m, \boldsymbol{p}_{2} m_{2}, \boldsymbol{P}_{d}-\boldsymbol{p}_{2} m_{3}> \\
& <\boldsymbol{p}_{2} m_{2}, \boldsymbol{P}_{d}-\boldsymbol{p}_{2} m_{3}\left|\Omega_{d}\right| \boldsymbol{P}_{d} \mathcal{M}_{d}>.
\end{aligned}
$$

The argument of the $N N$-matrix is defined as the threenucleon on-shell energy excluding the energy of the nucleon which does not participate in the interaction:

$$
E=E_{d}+E_{p}-E_{2}, \quad E^{\prime}=E_{d}+E_{p}-E_{3}^{\prime} .
$$

The superscript at the t-matrix in Eqs.(12), (18) refers to the isotopic momentum of the nucleon-nucleon pair.

\section{$3 \Delta$-isobar contribution.}

The structure of the amplitude with $\Delta$ in the intermediate state looks like the double-scattering one. But here we have $N N \rightarrow \Delta N$ matrixes instead of the nucleon-nucleon matrixes and $N N \Delta$-propagator instead of the three-nucleon one.

$$
\begin{aligned}
& \mathcal{J}_{\Delta}=2 \int d \boldsymbol{p}_{\mathbf{2}} d \boldsymbol{p}_{\mathbf{3}}^{\prime} d E_{\Delta} d \boldsymbol{p}_{\boldsymbol{\Delta}} \delta\left(E_{\Delta}-\sqrt{\mu^{2}+\boldsymbol{p}_{\boldsymbol{\Delta}}^{\mathbf{2}}}\right) \\
& \delta\left(\boldsymbol{p}+\boldsymbol{P}_{\boldsymbol{d}}-\boldsymbol{p}_{\mathbf{2}}-\boldsymbol{p}_{\mathbf{3}}-\boldsymbol{p}_{\boldsymbol{\Delta}}\right) \\
& 1<\left.\frac{1}{2} \tau^{\prime} \frac{1}{2} m^{\prime} \boldsymbol{p}^{\prime}\right|_{23}<00 ;-\boldsymbol{P}_{\boldsymbol{d}} 1 \mathcal{M}_{d}^{\prime}\left|\Omega_{d}^{\dagger}\left[1-P_{12}\right]\right| t_{3(N \Delta)}\left(E^{\prime}\right)
\end{aligned}
$$$$
\frac{1}{E-E_{2}-E_{3}^{\prime}-E_{\Delta}-i \Gamma\left(E_{\Delta} / 2\right)} \mid \Psi_{p_{\Delta}}\left(E_{\Delta}\right)>_{1}
$$$$
\mid \frac{1}{2} \tau_{2} \frac{1}{2} m_{2} \boldsymbol{p}_{2} ; \frac{1}{2} \tau_{3} \frac{1}{2} m_{3} \boldsymbol{p}_{3}>_{23}
$$$$
{ }_{23}<\frac{1}{2} \tau_{2} \frac{1}{2} m_{2} \boldsymbol{p}_{2} ;\left.\frac{1}{2} \tau_{3} \frac{1}{2} m_{3} \boldsymbol{p}_{3}\right|_{1}<\Psi_{\boldsymbol{p}_{\Delta}}\left(E_{\Delta}\right) \mid
$$$$
t_{2(N \Delta)}(E)\left[1-P_{13}\right] \Omega_{d}\left|\boldsymbol{P}_{\boldsymbol{d}} 1 \mathcal{M}_{d} ; 00>_{23}\right| \frac{1}{2} \tau \frac{1}{2} m \boldsymbol{p}>_{1}
$$

Moreover, the $\Delta$-isobar does not have a fixed mass and is defined by the mass-distribution. Therefore, we save here the integration over the $\Delta$-energy, $E_{\Delta}$. A full set of the particles quantum numbers was included into the amplitude definition in Eq.(20). Isospin and spin quantum numbers are marked by $\tau$ and $m$ or $\mathcal{M}_{d}$, respectively. The indexes near the bracket correspond to the particles numbers.

The distribution function of the $\Delta$-energy

$$
\left|\Psi_{p_{\Delta}}\left(E_{\Delta}\right)><\Psi_{p_{\Delta}}\left(E_{\Delta}\right)\right|=\rho\left(E_{\Delta}\right)
$$




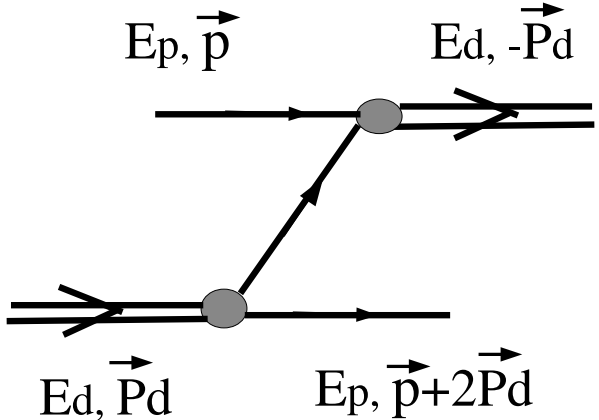

(a)

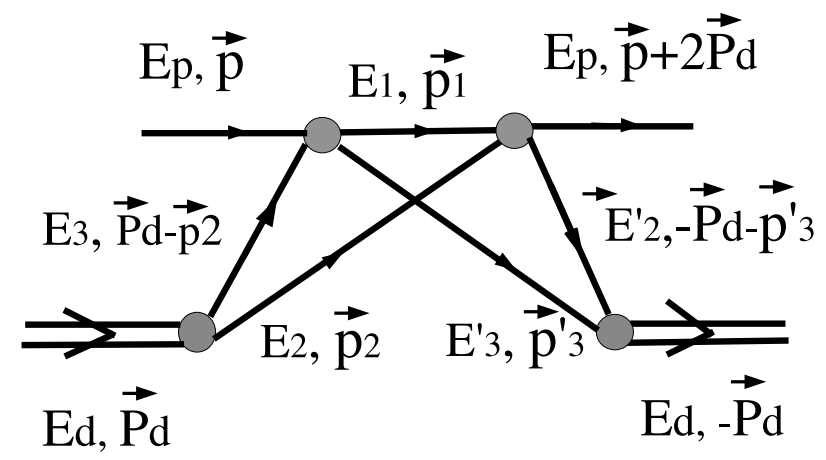

(c)

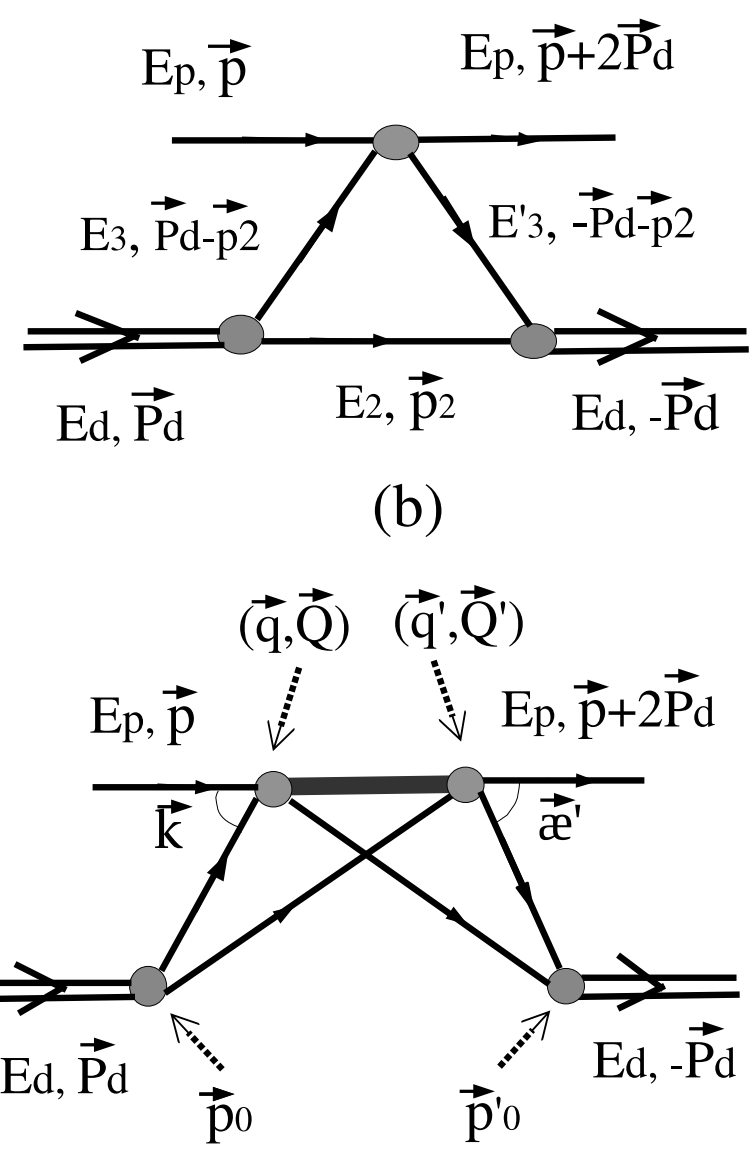

(d)

Fig. 1. The diagrams included into consideration: (a) the one nucleon exchange diagram; (b) the single scattering diagram; (c) the double scattering diagram with a nucleon in the intermediate state; (d)the double scattering diagram with $\Delta$-isobar in the intermediate state.

is defined through the $\Delta$-width $\Gamma(\mu)$ :

$$
\rho(\mu)=\frac{1}{2 \pi} \frac{\Gamma(\mu)}{\left(E_{\Delta}(\mu)-E_{\Delta}\left(m_{\Delta}\right)\right)^{2}+\Gamma^{2}(\mu) / 4},
$$

where $\mu^{2}=E_{\Delta}^{2}-\boldsymbol{p}_{\Delta}^{2}$ is a squared current mass of the delta. The delta width is energy dependent. We use, here, a standard parameterization of $\Gamma(\mu)$ taking into account the $\Delta$ off-shell corrections:

$$
\Gamma(\mu)=\Gamma_{0} \frac{p^{3}\left(\mu^{2}, m_{\pi}^{2}\right)}{p^{3}\left(m_{\Delta}^{2}, m_{\pi}^{2}\right)} \cdot \frac{p^{2}\left(m_{\Delta}^{2}, m_{\pi}^{2}\right)+\gamma^{2}}{p^{2}\left(\mu^{2}, m_{\pi}^{2}\right)+\gamma^{2}} .
$$

where $p\left(x^{2}, m_{\pi}^{2}\right)$ is the momentum value in the $\pi N$-centerof-mass:

$$
p\left(x^{2}, m_{\pi}^{2}\right)=\sqrt{\left(x^{2}+m_{N}^{2}-m_{\pi}^{2}\right)^{2} / 4 x^{2}-m_{N}^{2}} .
$$

All parameters were taken from ref. [37]:

$$
\Gamma_{0}=0.120 \mathrm{GeV}, \quad \gamma=0.200 \mathrm{GeV}, m_{\Delta}=1.232
$$

In the Born approximation the $N N \rightarrow N \Delta$ t-matrix can be replaced with the corresponding potential $V_{(N \Delta)}(E)$ :

$$
\begin{aligned}
& <\boldsymbol{p}, \frac{1}{2} m, \frac{1}{2} \tau\left|t_{(N \Delta)}(E)\right| \Psi_{\boldsymbol{p}_{\Delta}}\left(E_{\Delta}\right)>\approx \\
& <\boldsymbol{p}, \frac{1}{2} m, \frac{1}{2} \tau\left|V_{(N \Delta)}(E)\right| \Psi_{\boldsymbol{p}_{\Delta}}\left(E_{\Delta}\right)>
\end{aligned}
$$

The potential for the $N N \rightarrow N \Delta$ transition is based on the $\pi-$ and $\rho$ - exchanges:

$\begin{aligned} V_{\beta \alpha}^{(\pi)} & =-\frac{f_{\pi} f_{\pi}^{*}}{m_{\pi}^{2}} F_{\pi}^{2}(t) \frac{q^{2}}{m_{\pi}^{2}-t}(\boldsymbol{\sigma} \cdot \hat{q})(\boldsymbol{S} \cdot \hat{q})(\boldsymbol{\tau} \cdot \boldsymbol{T}) \\ V_{\beta \alpha}^{(\rho)} & =-\frac{f_{\rho} f_{\rho}^{*}}{m_{\rho}^{2}} F_{\rho}^{2}(t) \frac{q^{2}}{m_{\rho}^{2}-t}\{(\boldsymbol{\sigma} \boldsymbol{S})-(\boldsymbol{\sigma} \cdot \hat{q})(\boldsymbol{S} \cdot \hat{q})\}(\boldsymbol{\tau} \cdot \boldsymbol{T})\end{aligned}$

Here, $t$ is a four-momentum transfer and $\boldsymbol{q}$ is a corresponding three-momentum transfer. Operators $\boldsymbol{\sigma}(\boldsymbol{\tau})$ are $\frac{1}{2}$ - spin (isospin) operators defined by Pauli matrixes while $\boldsymbol{S}(\boldsymbol{T})$ operators correspond to $\frac{1}{2} \rightarrow \frac{3}{2}$ spin (isospin) transition. $m_{\pi}$ and $m_{\rho}$ are pion and $\rho$ - meson masses. The coupling 


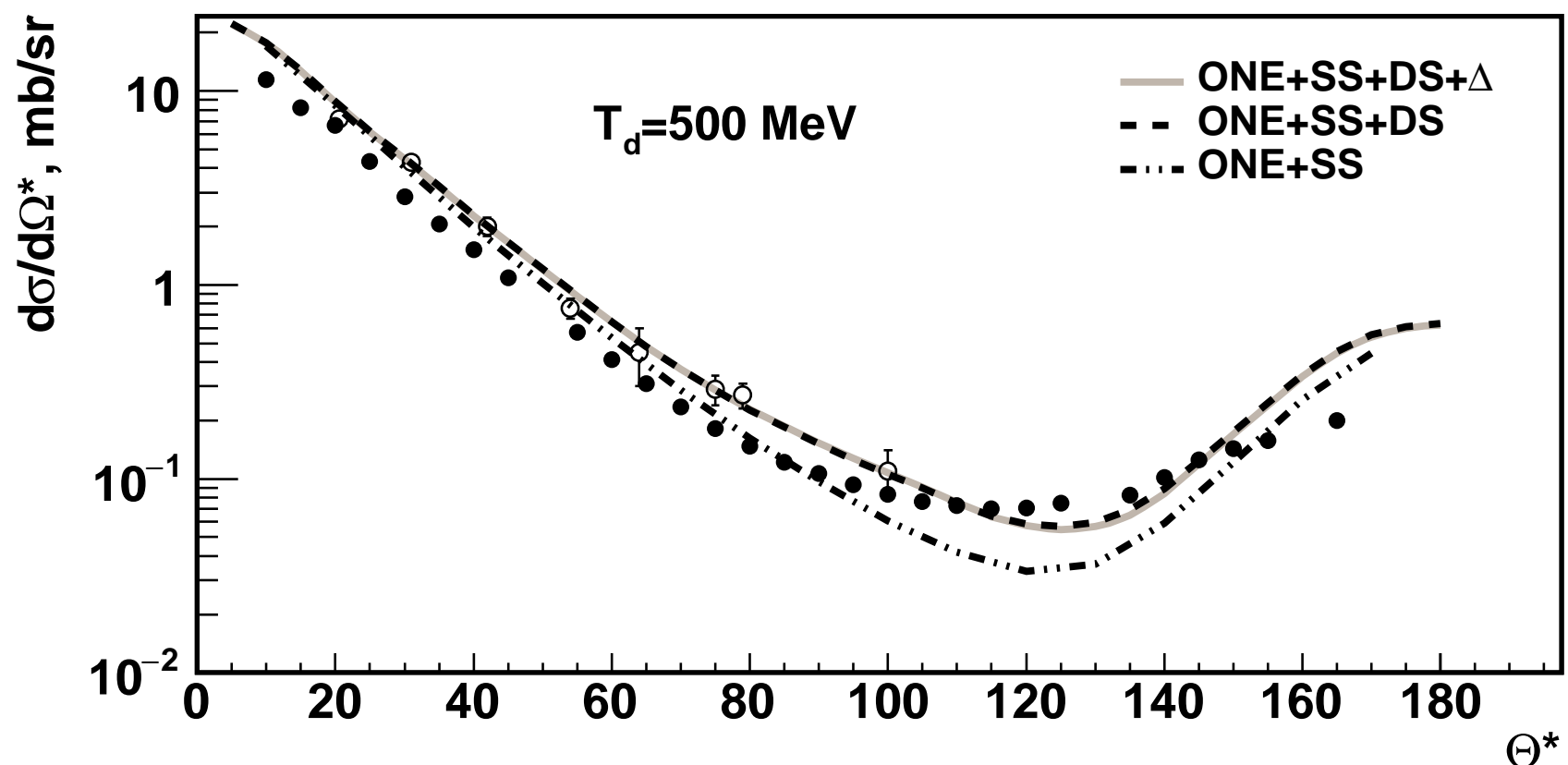

Fig. 2. The differential cross section at the deuteron kinetic energy of $500 \mathrm{MeV}$ as a function of the c.m. scattering angle. The data are taken from[16] $(\bullet)$, and 1 (o).

constant $f_{\pi}$ is related with the $N N \pi$ vertex and $f_{\pi}^{*}$ corresponds to the $N \Delta \pi$ one. It also concerns $\rho$ - coupling constants.

$$
\begin{array}{lrl}
f_{\pi}=1.008 & f_{\pi}^{*}=2.156 \\
f_{\rho}=7.8 & f_{\rho}^{*}=1.85 f_{\rho}
\end{array}
$$

The hadronic form factor was chosen in the monopole form:

$$
F_{x}(t)=\left(\Lambda_{x}^{2}-m_{x}^{2}\right) /\left(\Lambda_{x}^{2}-t\right)
$$

In our calculation we use $\Lambda=1 \mathrm{GeV}$.

The presented approach for the $\Delta$-isobar term was applied in refs. 37, 38, to describe the data on the $\Delta$ - production in the $p p \rightarrow n \Delta^{++}$. The obtained results were in a good agreement with the experimental data on the differential cross section.

Since two nucleon states in the $N N \rightarrow N \Delta$ vertexes are antisymmetrized, two permutation operators appear in Eq.(20). As consequence, the $\Delta$ - amplitude contains four terms: one direct, two exchange, and one doubleexchange ones. The permutation operator $P_{i j}$ involves the permutation of all quantum numbers. Here, it is permutation over momentum, spin, and isospin indexes: $P_{i j}=$ $P_{i j}(p) P_{i j}(\sigma) P_{i j}(\tau)$.

The isotopic coefficient in Eq.(20) is equal for the all four contributions into the $\Delta$ - amplitude and defined as

$$
\begin{aligned}
c_{T}= & <\frac{1}{2} \tau_{2}^{\prime} \frac{1}{2} \tau_{3}^{\prime}\left|00><\frac{1}{2} \tau^{\prime} \frac{1}{2} \tau_{2}^{\prime}\right|\left(\boldsymbol{T}_{1} \boldsymbol{\tau}_{2}\right) \mid \frac{3}{2} \tau_{\Delta} \frac{1}{2} \tau_{2}> \\
& <\frac{3}{2} \tau_{\Delta} \frac{1}{2} \tau_{3}^{\prime}\left|\left(\boldsymbol{T}_{1} \boldsymbol{\tau}_{3}\right)\right| \frac{1}{2} \tau \frac{1}{2} \tau_{3}><\frac{1}{2} \tau_{2} \frac{1}{2} \tau_{3} \mid 00>.
\end{aligned}
$$

It can be calculated independently on the spin part of the $\Delta$ - amplitude:

$$
c_{T}=\frac{1}{12}<\frac{1}{2}\|T\| \frac{3}{2}>^{2}<\frac{1}{2}\|\tau\| \frac{1}{2}>^{2}=2,
$$

where the reduced matrix elements $<\frac{3}{2}\|T\| \frac{1}{2}>=$

$<\frac{3}{2}\|S\| \frac{1}{2}>=2$ and $<\frac{1}{2}\|\tau\| \frac{1}{2}>=<\frac{1}{2}\|\sigma\| \frac{1}{2}>=\sqrt{6}$ were substituted.

The permutation over spin states is defined in the same way it was done for the one-nucleon exchange term (8).

\subsection{Kinematics}

We consider, here, the kinematics of the double scattering diagram in detail. All the presented expressions concern the diagram with a $\Delta$ - excitation. However, they are also correct for the double-scattering diagram with a nucleon in the intermediate state, if $\left(E_{\Delta}, \boldsymbol{p}_{\Delta}\right)$ is replaced with $\left(E_{1}, \boldsymbol{p}_{1}\right)$. The momenta notations are shown in Fig.(1c).

The two nucleons relative momenta in the deuterons vertexes are defined in relativistic kinematics in the following manner:

$$
\begin{aligned}
& \boldsymbol{p}_{\mathbf{0}}=\frac{\left(E_{2}+E^{*}\right) \boldsymbol{p}_{3}-\left(E_{3}+E^{*}\right) \boldsymbol{p}_{2}}{E_{2}+E_{3}+2 E^{*}} \\
& \boldsymbol{p}_{\mathbf{0}}^{\prime}=\frac{\left(E_{2}^{\prime}+E^{\prime *}\right) \boldsymbol{p}_{3}^{\prime}-\left(E_{3}^{\prime}+E^{\prime *}\right) \boldsymbol{p}_{2}^{\prime}}{E_{2}^{\prime}+E_{3}^{\prime}+2 E^{\prime *}} .
\end{aligned}
$$

Here, $E^{*}$ and $E^{\prime *}$ are the energies one of the nucleon in the corresponding deuteron rest frame, which is equivalent 


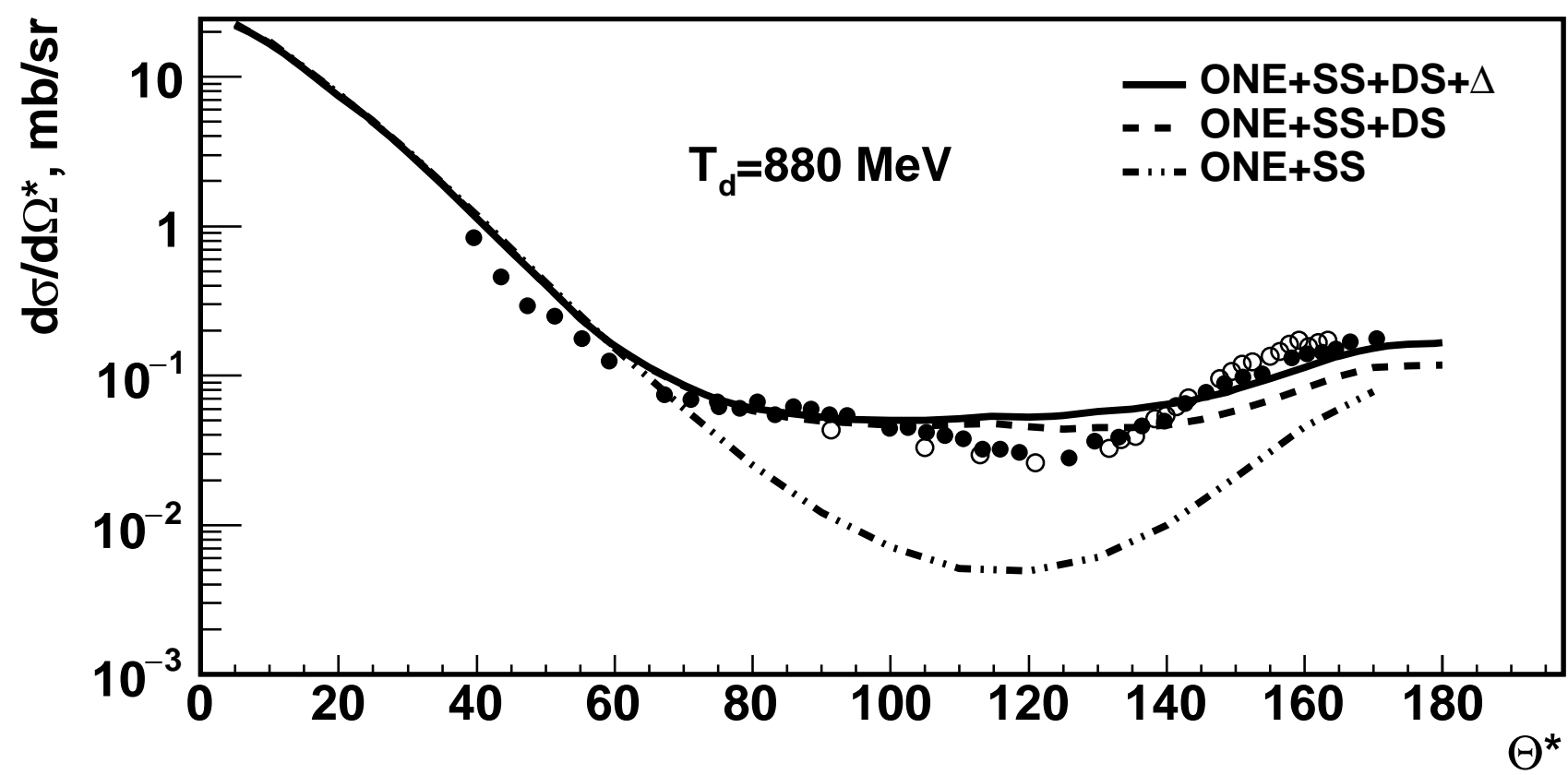

Fig. 3. The differential cross section at the deuteron kinetic energy of $880 \mathrm{MeV}$ as a function of the c.m. scattering angle. The data are taken from 39] $(\bullet)$, and 40](०).

the two nucleons centre-of-mass:

$$
E^{*}=\sqrt{\left(p_{2}+p_{3}\right)^{2}} / 2, \quad E^{* *}=\sqrt{\left(p_{2}^{\prime}+p_{3}^{\prime}\right)^{2}} / 2
$$

In accordance to the momentum conservation in the deuteron vertexes we can get the following expressions for the internal deuteron momenta in the deuterons Breit frame:

$$
\begin{aligned}
& \boldsymbol{p}_{\mathbf{0}}=\frac{\boldsymbol{P}_{d}}{2}-\boldsymbol{p}_{2}-\frac{E_{2}-E_{3}}{E_{2}+E_{3}+2 E^{*}} \frac{\boldsymbol{P}_{d}}{2} \\
& \boldsymbol{p}_{\mathbf{0}}^{\prime}=\frac{\boldsymbol{P}_{d}}{2}+\boldsymbol{p}_{3}^{\prime}+\frac{E_{2}^{\prime}-E_{3}^{\prime}}{E_{2}^{\prime}+E_{3}^{\prime}+2 E^{\prime *}} \frac{\boldsymbol{P}_{d}}{2}
\end{aligned}
$$

Note, that two first terms in these equations correspond to the ordinary definition of the nonrelativistic relative momentum.

The transfer momenta in the $N N \rightarrow \Delta N$ vertexes, $\boldsymbol{q}$ and $\boldsymbol{q}^{\prime}$, are defined in a standard manner:

$$
\boldsymbol{q}=\frac{1}{2}\left(\boldsymbol{k}^{\prime}-\boldsymbol{k}\right) \quad \text { and } \quad \boldsymbol{q}^{\prime}=\frac{1}{2}\left(\varkappa^{\prime}-\varkappa\right),
$$

where vectors $\boldsymbol{k}, \boldsymbol{k}^{\prime}$ are the nucleon-nucleon and nucleondelta relative momenta in the $N N \rightarrow \Delta N$ vertex :

$$
\begin{gathered}
\boldsymbol{k}=\frac{\left(E_{3}+E_{13}^{*}\right) \boldsymbol{p}-\left(E_{p}+E_{13}^{*}\right) \boldsymbol{p}_{3}}{E_{p}+E_{3}+2 E_{13}^{*}} \\
\boldsymbol{k}^{\prime}=\frac{\left(E_{3}^{\prime}+E_{13}^{\prime *}\right) \boldsymbol{p}_{\Delta}-\left(E_{\Delta}+E_{13}^{\prime *}\right) \boldsymbol{p}_{3}^{\prime}}{E_{\Delta}+E_{3}^{\prime}+2 E_{13}^{\prime *}}
\end{gathered}
$$

and $\varkappa, \varkappa^{\prime}$ are the nucleon-delta and nucleon-nucleon relative momenta in the $\Delta N \rightarrow N N$ vertex :

$$
\begin{gathered}
\varkappa=\frac{\left(E_{2}+E_{12}^{*}\right) \boldsymbol{p}_{\Delta}-\left(E_{\Delta}+E_{12}^{*}\right) \boldsymbol{p}_{2}}{E_{\Delta}+E_{2}+2 E_{12}^{*}} \\
\varkappa^{\prime}=\frac{\left(E_{2}^{\prime}+E_{12}^{\prime *}\right) \boldsymbol{p}^{\prime}-\left(E_{p}+E_{12}^{\prime *}\right) \boldsymbol{p}_{2}^{\prime}}{E_{p}+E_{2}^{\prime}+2 E_{12}^{\prime *}} .
\end{gathered}
$$

$E_{i j}^{*}=\sqrt{\left(p_{i}+p_{j}\right)^{2}} / 2$ is the invariant variable defined by $p_{i}$ and $p_{j}$ four-momenta.

In this section we considered only a direct term in the amplitude with the delta in the intermediate state. The other terms can be obtained by the replacement the corresponding momentum with one with an opposite sign. In such a way $\boldsymbol{q}=\left(\boldsymbol{k}^{\prime}-\boldsymbol{k}\right) / 2$, for example, replaces with $\boldsymbol{Q}=\left(\boldsymbol{k}^{\prime}+\boldsymbol{k}\right) / 2$ etc.

\subsection{Spin part}

To simplify the calculation in this paper we consider the reduced $N \rightarrow \Delta$ t-matrix. The $\rho$-meson part is not taken into account. We believe that the $\pi$-meson term gives the general contribution into the differential cross section at backward angles. However, $\rho$-meson can be very important to describe some polarisation observables due to its vector nature. Therefore, it will be included into consideration in further calculations.

In such a way spin structure of the $\Delta$-amplitude is defined by the expression:

$$
(23)<1 \mathcal{M}^{\prime} \mid u\left(p_{0}^{\prime}\right)+
$$




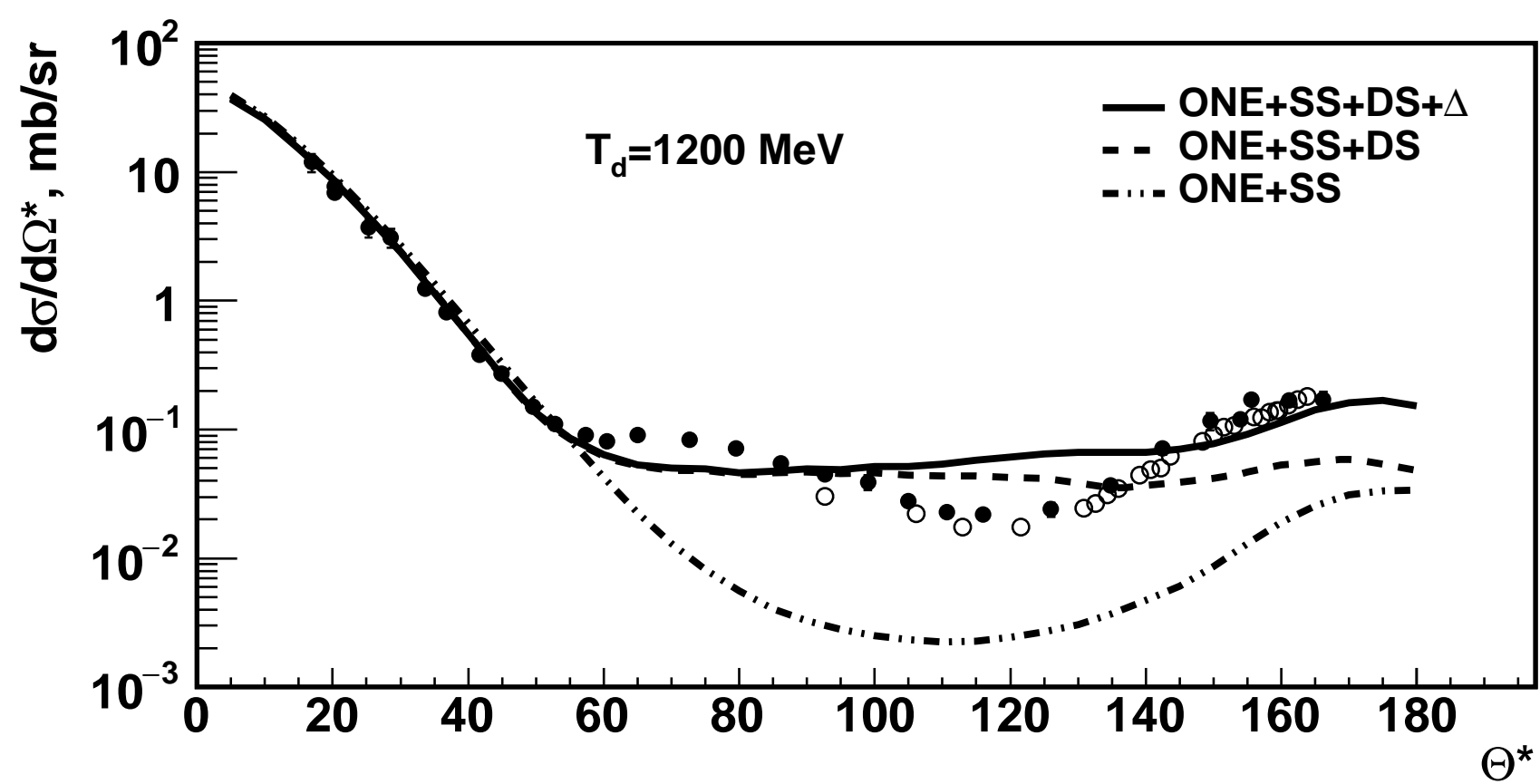

Fig. 4. The differential cross section at the deuteron kinetic energy of $1200 \mathrm{MeV}$ as a function of the c.m. scattering angle. The data are taken from 41] (•), and 40, (०).

$$
\begin{aligned}
& \frac{w\left(p_{0}^{\prime}\right)}{\sqrt{2}}\left[3\left(\boldsymbol{\sigma}_{2} \hat{p_{0}^{\prime}}\right)\left(\boldsymbol{\sigma}_{3} \hat{p_{0}^{\prime}}\right)-\left(\boldsymbol{\sigma}_{2} \boldsymbol{\sigma}_{3}\right)\right] \mid \frac{1}{2} m_{2}^{\prime} \frac{1}{2} m_{3}^{\prime}> \\
& <\frac{1}{2} m_{2}^{\prime}\left|\left(\boldsymbol{\sigma}_{2} \hat{q^{\prime}}\right)\right| \frac{1}{2} m_{2}><\frac{1}{2} m_{3}^{\prime}\left|\left(\boldsymbol{\sigma}_{3} \hat{q}\right)\right| \frac{1}{2} m_{3}> \\
& <\frac{1}{2} m_{2} \frac{1}{2} m_{3} \mid u\left(p_{0}\right)+ \\
& \frac{w\left(p_{0}\right)}{\sqrt{2}}\left[3\left(\boldsymbol{\sigma}_{2} \hat{p_{0}}\right)\left(\boldsymbol{\sigma}_{3} \hat{p_{0}}\right)-\left(\boldsymbol{\sigma}_{2} \boldsymbol{\sigma}_{3}\right)\right] \mid 1 \mathcal{M}>_{(23)} \\
& <\frac{1}{2} m^{\prime}\left|\left(\boldsymbol{S}_{1} \hat{q^{\prime}}\right)\right| \frac{3}{2} M_{\Delta}><\frac{3}{2} M_{\Delta}\left|\left(\boldsymbol{S}_{1} \hat{q}\right)\right| \frac{1}{2} m>.
\end{aligned}
$$

After straightforward calculations we get some combinations of $\sigma_{1, i}, \sigma_{1, i} \sigma_{2, j}$ and etc. sandwiched between deuterons spin states. It is useful to apply the following relations:

$$
\begin{aligned}
& (23)<1 \mathcal{M}^{\prime}\left|\boldsymbol{\sigma}_{2, i}\right| 1 \mathcal{M}>_{(23)}= \\
& (23)<1 \mathcal{M}^{\prime}\left|\boldsymbol{\sigma}_{3, i}\right| 1 \mathcal{M}>_{(23)}= \\
& (23)<1 \mathcal{M}^{\prime}\left|\boldsymbol{S}_{d, i}\right| 1 \mathcal{M}>_{(23)} \\
& (23)<1 \mathcal{M}^{\prime}\left|\boldsymbol{\sigma}_{2, i} \boldsymbol{\sigma}_{3, j}\right| 1 \mathcal{M}>_{(23)}>= \\
& <1 \mathcal{M}^{\prime}\left|\frac{1}{3} \delta_{i j}+2 Q_{i j}\right| 1 \mathcal{M}>_{(23)},
\end{aligned}
$$

where $\boldsymbol{S}_{d}$ and $Q_{i j}$ are the spin and tensor operators of a deuteron, respectively. Also the following expression

$$
\begin{aligned}
& <\frac{1}{2} m^{\prime}\left|\left(\boldsymbol{S} \hat{q^{\prime}}\right)\right| \frac{3}{2} M_{\Delta}><\frac{3}{2} M_{\Delta}|(\boldsymbol{S} \hat{q})| \frac{1}{2} m>= \\
& \frac{1}{6}<\frac{1}{2}\|S\| \frac{3}{2}>^{2}\left[\left(\hat{q} \hat{q^{\prime}}\right)<\frac{1}{2} m^{\prime}|I| \frac{1}{2} m>+\right.
\end{aligned}
$$

$$
\left.\frac{i}{2}<\frac{1}{2} m^{\prime}\left|\left(\boldsymbol{\sigma} \hat{q} \times \hat{q}^{\prime}\right)\right| \frac{1}{2} m>\right]
$$

is very helpful to simplify the calculations with $3 / 2$-spin operators.

\section{Results and discussions.}

We applied the presented above model to describe the differential cross sections. The formal definition of the total cross section of deuteron-proton elastic scattering is given by

$$
\begin{aligned}
& \sigma(d p \rightarrow d p)=(2 \pi)^{4} \frac{1}{6} \int \frac{d \boldsymbol{P}_{d}^{\prime}}{E_{d}^{\prime}} \frac{d \boldsymbol{p}^{\prime}}{E_{p}^{\prime}} \delta\left(E_{d}+E_{p}-E_{d}^{\prime}-E_{p}^{\prime}\right) \\
& \delta\left(\boldsymbol{P}_{d}+\boldsymbol{p}-\boldsymbol{P}_{d}^{\prime}-\boldsymbol{p}^{\prime}\right) \frac{|T|^{2}}{\sqrt{\left(P_{d} p\right)^{2}-P_{d}^{2} p^{2}}},
\end{aligned}
$$

where $|T|^{2}$ is the squared invariant amplitude defined as

$$
|T|^{2}=\left|\sqrt{E_{d}^{\prime} E_{p}^{\prime}} \mathcal{J}_{d p \rightarrow d p} \sqrt{E_{d} E_{p}}\right|^{2} \equiv i n v .
$$

The expression for the differential cross section in the center-of-mass can be obtained through the scattering amplitude in the Breit frame as:

$\frac{d \sigma}{d \cos \theta^{*}}=\frac{(2 \pi)^{5}}{24 s}\left(s-m_{N}^{2}-M_{d}^{2}+\frac{t}{2}\right)^{2}\left|\mathcal{J}_{d p \rightarrow d p}^{(\text {Breit })}(s, t)\right|^{2}$,

where $s=\left(P_{d}+p\right)^{2}$ and $t=\left(p-p^{\prime}\right)^{2}$ are invariant Mandelstam variables. 


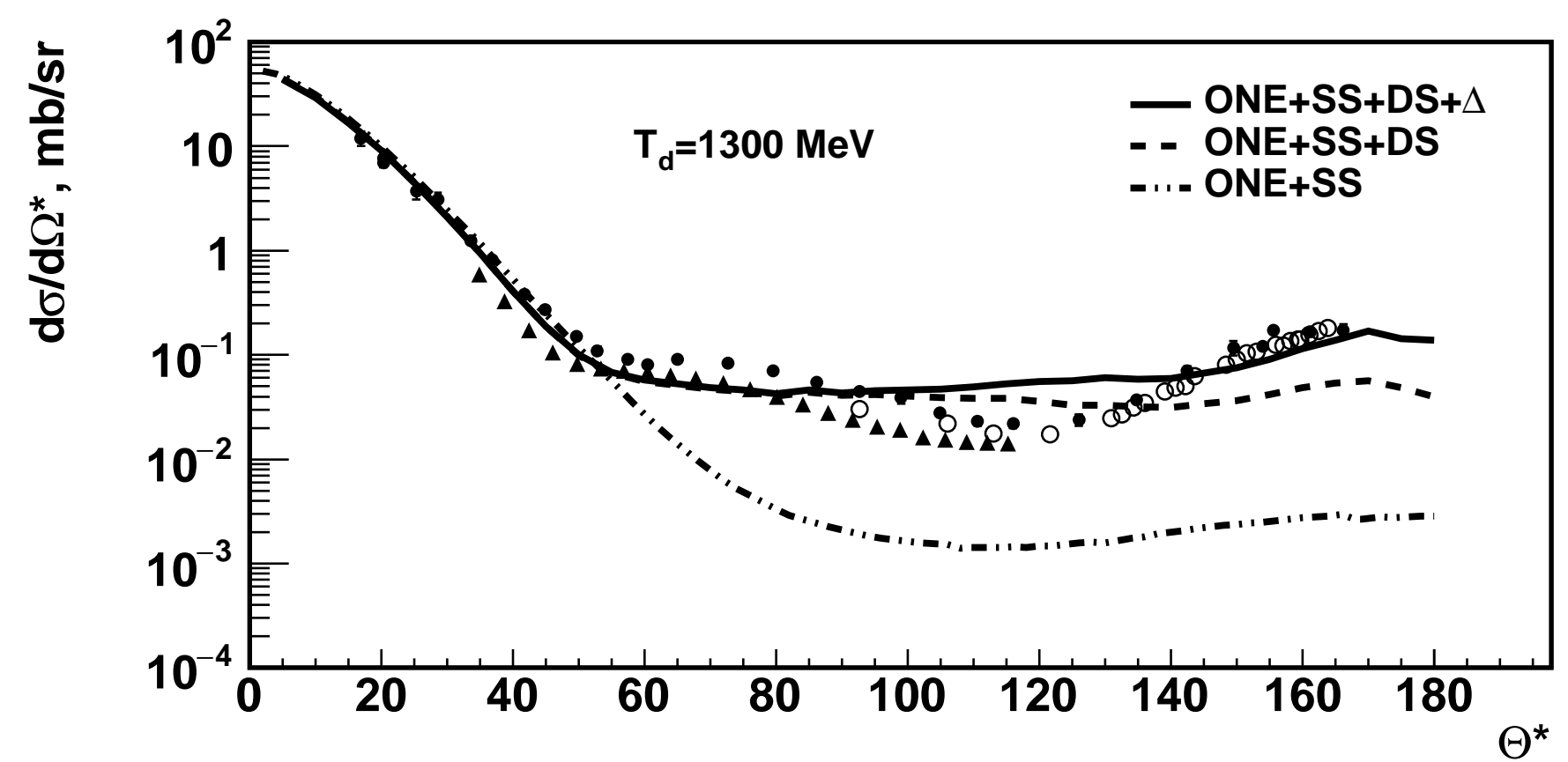

Fig. 5. The differential cross section at the deuteron kinetic energy of $1300 \mathrm{MeV}$ as a function of the c.m. scattering angle. The data are taken for $T_{d}=1200 \mathrm{MeV}$ from 41 ] $(\bullet), T_{d}=1180 \mathrm{MeV}$ [40] (०), and $T_{d}=1282 \mathrm{MeV}$ [2] $(\boldsymbol{\Delta})$.

We evaluated the dp-elastic differential cross section at four energies in a wide range. The results presented for three cases: 1) only one-nucleon exchange (ONE) and single-scattering (SS) diagrams taken into account, 2) the double-scattering (DS) term is added into the consideration, and 3 ) the $\Delta$-isobar excitation is included.

At first, the deuteron kinetic energy equal to $500 \mathrm{MeV}$ was considered (Fig 2). One can see, the SS-diagram plays an important role up to the scattering angle equal to about $60^{\circ}$. The DS-contribution is significant at the scattering angle between $60^{\circ}$ and $160^{\circ}$. The difference between the results taking into account the double scattering and without it reaches $2-3$ times at $\theta^{*}=120^{\circ}$.

This energy is not high enough for the $\Delta$-isobar manifestation. Therefore, the inclusion of the $\Delta$-contribution term into the scattering amplitude does not have to influence remarkably to the result. In fact, the results obtained with and without the $\Delta$-isobar term are undistinguished in a whole angular range.

The second energy is equal to $880 \mathrm{MeV}$ (Fig 3). One can see, the contribution of the double-scattering is very significant at this energy. The inclusion of the DS-term into consideration allows to describe the behaviour of the differential cross section at the scattering angle range $60^{\circ}-$ $140^{\circ}$. The difference between $\mathrm{ONE}+\mathrm{SS}$ curve and $\mathrm{ONE}+$ $\mathrm{SS}+\mathrm{DS}$ one reaches about 15 times. Note, the $\Delta$ excitation begins to manifest itself at the angle equal to about $120^{\circ}$ and describes the behaviour of the experimental data at the angle above $140^{\circ}$ where the differential cross section sharply increases.
The dp-elastic differential cross sections at energies equal to $T_{d}=1200 \mathrm{MeV}$ and $T_{d}=1300 \mathrm{MeV}$ are presented in Fig 4 and Fig [5, respectively. Here we can observe further enhancement of the double scattering term influence on the result. Also the contribution of the $\Delta$ isobar increases significantly at the scattering angle above $140^{\circ}$.

Analysing the obtained results one can conclude the following. The differential cross sections are described quite well up to the scattering angles equal to $60^{\circ}$ taking into account only the one-nucleon-exchange and single-scattering terms. If we consider the dp-elastic scattering at the angles large than $60^{\circ}$, it is necessary to include the double scattering term into consideration. It should be noted the double-scattering contribution into the reaction amplitude increases with the deuteron energy growing and may change the value of the differential cross section on a few orders in comparison with the result obtained without inclusion of the DS-term.

The sharp rise of the differential cross section at $\theta^{*} \geq$ $140^{\circ}$, which is observed in the experimental data, points at the appearance of the addition reaction mechanism. The inclusion of the $\Delta$-excitation term into consideration allowed to describe this rise of the differential cross section at the large scattering angles. The contribution of the $\Delta$ isobar mechanism grows with the initial deuteron energy. It is negligible at $T_{d}=500 \mathrm{MeV}$ and significant at $T_{d}=$ $1300 \mathrm{MeV}$.

In such way we got a good description of the differential cross section of deuteron-proton elastic scattering in a whole angular range, from $0^{\circ}$ to $180^{\circ}$ of the scattering an- 
gle, in a wide deuteron energy interval, between $500 \mathrm{MeV}$ and $1300 \mathrm{MeV}$.

Acknowledgements: The author is grateful to Dr. V.P. Ladygin for fruitful discussions and interest in this problem. This work has been supported by the Russian Foundation for Basic Research under grant Nํㅗ No.16-02-00203a.

\section{References}

1. R.D.Shamberger, Phys.Rev. 85, 424 (1952).

2. O.Chamberlain, M.O.Stern, Phys.Rev.94, (1954) 666

3. O.Chamberlain, D.D.Clark, Phys.Rev.102, (1956) 473

4. A.V.Crewe, et al., Phys.Rev.114, (1959) 1361

5. L.Marshall, et al., Phys.Rev.95, (1954) 1020

6. S.Marcowitz, Phys.Rev.120, (1960) 891

7. H.Postma, R.Wilson, Phys.Rev.121, (1961) 1229

8. G. Igo et al., Nucl.Phys.,A195, (1972) 33,

9. M.A. Nasser et al., Nucl.Phys., A229, (1974) 113

10. M.Garcon et al., Nucl.Phys.A 458, 287 (1986).

11. J.Arvieux et al., Nucl.Phys.A 431, 613 (1984).

12. M.Haji-Saied et al., Phys. Rev.C 36, 2010 (1987).

13. Sun Tsu-hsun et al. Phys.Rev.C 31515 (1985).

14. A.Rahbar et al. Rhys. Lett.B 194338 (1987).

15. V.Ghazikhanian et al. Phys.Rev.C 431532 (1991).

16. K.Hatanaka et al., Phys.Rev.C66, (2002) 044002

17. Y.Maeda et al., Phys.Rev.C, (2007) 014004

18. K.Ermish et al., Phys.Rev.C71, (2005) 064004

19. V.V. Glagolev, V.P. Ladygin, N.B. Ladygina, A.A. Terekhin, Eur.Phys.J. A48, (2012) 182

20. P.K.Kurilkin, et al., Phys.Lett.B 715, (2012) 61

21. N.B.Ladygina, Phys.Atom.Nucl.71, (2008) 2039 .

22. N.B.Ladygina, Eur. J. Phys. A 42, (2009) 91.

23. A.K.Kerman, L.S.Kisslinger, Phys.Rev. 180, 1483 (1969).

24. L.A.Kondratyuk, F.M.Lev, Sov.J.Nucl.Phys. 26, 153 (1977).

25. L.A.Kondratyuk, F.M.Lev, L.V.Shevchenko, Sov.J.Nucl.Phys. 29, 558 (1979).

26. L.A.Kondratyuk, F.M.Lev, L.V.Shevchenko, Yad.Fiz. 33, 1208 (1981).

27. A.Deltuva, K.Chmielewski, P.U.Sauer, Phys.Rev.C67, 034001 (2003).

28. A.Deltuva, R.Machleidt, P.U.Sauer, Phys.Rev.C68, 024005 (2003).

29. E.O.Alt, P.Grassberger, W.Sandhas, Nucl.Phys. B2, 167 (1967)

30. E.Schmid, H.Ziegelmann, The Quantum Mechanical ThreeBody Problem (Oxford, Pergamon Press, 1974).

31. R.Machleidt, K.Holinde, Ch.Elster, Phys.Rep. 149, 1 (1987).

32. R.Machleidt, Phys. Rev. C63, 024001 (2001).

33. M. Lacombe et al., Phys.Lett.B101, 139 (1981).

34. W.G.Love, M.A.Franey, Phys. Rev.C 24, 1073 (1981)

35. N.B.Ladygina (2008), e-preprint nucl-th/0805.3021

36. http://gwdac.phys.gwu.edu

37. B.K.Jain, A.B.Santra, Phys.Rep.230, 1 (1993)

38. V.Dmitriev, O.Sushkov, C.Gaarde, Nucl.Phys. A459, 503 (1986)

39. N.E.Booth et al., Phys.Rev.D 4, 1261 (1971).

40. J.C.Alder et al., Phys.Rev.C 6, 2010 (1972).

41. E.T.Boschitz et al., Phys.Rev.C 6, 457 (1972)

42. E.Gülmez et al.,Phys.Rev.C 42, 2067 (1991). 
